\title{
Hazard identification and pre-map with a simple specific tool: synthesis of application experience in handicrafts in various productive sectors
}

\author{
Daniela Colombini* ., Enrico Occhipinti**,Raffaele Peluso***, Loretta Montomoli**** \\ * EPM, Research Unit "Ergonomics of Posture and Movement", Don C. Gnocchi Foundation-Milan-Italy \\ ** EPM, Research Unit "Ergonomics of Posture and Movement", Policlinico Ca' Granda Foundation-Milan- \\ Italy \\ ***CESMEL -Pisa \\ ****Unit of Occupational Medicine, University of Siena
}

\begin{abstract}
In August 2009, an international group was founded with the task of developing a "toolkit for MSD prevention" under the IEA and in collaboration with the World Health Organization.

According to the ISO standard 11228 series and the new Draft ISO TR 12259 "Application document guides for the potential user", our group developed a preliminary "mapping" methodology of occupational hazards in the craft industry, supported by software (Excel®, free download on: www.epmresearch.org).

The possible users of toolkits are: members of health and safety committees; health and safety representatives; line supervisors; foremen; workers; government representatives; health workers providing basic occupational health services; occupational health and safety specialists.

The proposed methodology, using specific key enters and quick assessment criteria, allows a simple ergonomics hazards identification and risk estimation to be made. It is thus possible to decide for which occupational hazards a more exhaustive risk assessment will be necessary and which occupational consultant should be involved (occupational physician, safety engineer, industrial hygienist, etc.).

The methodology has been applied in different situations of small and medium craftsmanship Italian enterprises: leather goods, food, technical dental work, production of artistic ceramics and stained glass, beekeeping activities. The results are synthetically reported and discussed in this paper.
\end{abstract}

Key words: biomechanical overload, ergonomic risk assessment, preliminary mapping methodology

\section{Introduction}

During the last Congress of the International Ergonomics Association (IEA), Beijing, August 2009, an international group was founded with the task of developing a "toolkit for MSD prevention" under the IEA and in collaboration with the World Health Organization.

According to the ISO standard 11228 series $[6,7,8,9]$ and the new Draft CD ISO 12259-2009: Application document guides for the potential user 4]:, our group developed a preliminary "mapping" methodology of occupational hazards in the craft industry, supported by software (in Excel ${ }^{\circledR}$,).freely download on: www.epmresearch.org: en_PREMAPPING_ERGOCHECK_18-7-11q

The methodology can be summarized in the following three points:

-global : global interpretation of worker's discomfort be it originated from work place or work environment.

-simple: the methodology materializes into an easily usable information collection model. For this 
descriptive stage, computer-based closed-reply questions are used (Excel®).

- priority scales for subsequent assessment fulfilments: the results (automatically achieved from software and easily readable through summarizing histograms) will help identify problems as well as provide a priority scale for subsequent assessment fulfilments.

The possible users of toolkits are: members of health and safety committees; health and safety representatives; line supervisors; labour inspectors; occupational health and safety specialists (doctors and technicians, etc.).

The methodology has been applied in different situations of small and medium craftsmanship Italian enterprises: leather goods, food, technical dental work, production of artistic ceramics and stained glass, beekeeping activities.

\section{Methods}

The first (basic level) using key-enters techniques involves a quick and overall identification of possible risk inducers through specific key enters. This basic level is subdivided into a variety of "frames" for each type of risk: load manual handling, upper limb repetitive movements, postures, noise, microclimate, chemical agents, work organization, etc..

The subsequent intervention level using quick assessment techniques involves quick identification of full risk acceptable conditions (marked, with the traditional traffic light system, by green code or very high risk conditions (critical code).

For green code the risk assessment process could also interrupt at this level since the working risk turns out to be acceptable. For critical code situations, the risk is certainly present and high: it is however necessary to proceed with a more in-depth risk assessment using reference tools and standards currently available asking for a quick improvement action. Both the green code green and critical code can be identify trough pre-established scenarios, describing the activities developed by the different homogeneous groups.

If after quick assessment the workplace is neither at negligible risk (green code) nor in clearly critical conditions, the risk has to be analytically assessed through risk analysis models as proposed by ISO standards.

Methodology materializes into an easily usable information collection model already computer based en_PREMAPPING_ERGOCHECK 18-711.xls), that can be freely downloaded www.epmresearch.org. Let us now go into a synthetical description of the pre-mapping model. For more detailed information see the paper presented by the authors in another special symposium (Relevant contributions for a IEA/WHO toolkit devoted to MSD prevention) of this IEA 2012 Conference ("A simple tool for preliminary hazard identification and quick assessment in craftwork and small/medium enterprises by Colombini et al). For duty space problem, we cannot show the contents of all the key-enter indicators for the hazard identification of all the risk analyzed in the presented proposal: as suggested before, we recommended to see the model proposed in software en_PREMAPPING_ERGOCHECK_18-7-11.

Intentionally these preliminary indicators omit the scores but use only a chromatic priority scales easily expressing on one side the absence of the problem and on the other the need to tackle urgently. Here we have to limit to list the titles of the risk involved in this kinds of evaluation:

\subsection{Identification data (ID) and basic intervention level through key-enters}

- ID data of enterprise After outlining the enterprise, pre-mapping form filling is addressed to one worker or one group of workers (homogeneous group) with the same job and tasks in the shift.

- Key-enters for identification of assessment priorities for biomechanical overload risks (Figure 1), the same as proposed by ISO 11228 standards 1-2-and 3 and ISO 11226 and all in the news presented in CD ISO 12259 DRAFT in preparation. Key-enters are quite simple questions that can be answered by a YES/NO reply.

- Key-enters for identification of interior lighting problems.

- Key-enters for identifying outside work-related problems - UV radiations.

- Key-enters for identifying noise-related problems.

- Key-enters for identifying problems associated with microclimate.

- Key-enters for identifying problems associated with use of equipment/tools.

- Key-enters for identifying problems associated with vibration exposure. 
- Key-enters for identifying problems associated with use of machinery or parts off machinery.

- Key-enters for identifying problems from polluting agents (chemical hazard, biological hazard) and other particular risk factors

- Key-enters for identifying organizational problems.

- Key-enters for identifying problems generically associated with potential stress inductors

\subsection{The first action level by quick assessment}

Once possible (absent/present) risk inductors have been identified through key-enters, we can proceed with quick identification of risk being absent or high, through simplified assessment techniques such as quick assessment. This procedure does not use calculation schemes or formulas but deals with 3 different fronts:

- recording of critical conditions: the critical codes (purple light)

- recording of acceptability conditions: the green codes (green light)

- if recorded conditions are neither critical nor acceptable, it is necessary to proceed with traditional risk assessment methodologies as provided by reference standards (ISO and CEN) and in the ISO Technical Report (DIS ISO/TR 12259- Application document for ISO 11228 series)

For the other physical risks listed in the keyenters sections, other more experienced group could work out in the future for developing specific quickassessment techniques, if possible. Only as to chemical hazard indicators, a detailed sheet is proposed: it allows a more detailed investigation of hazardous situations in order to define the more or less urgent need to complete the assessment procedure.

The quick assessment models have the following parts:

- Quick assessment of repetitive tasks.

- Quick-assessment of load manual handling.

- Quick assessment of load manual carrying.

- Quick assessment of load pulling and/or pushing

- Description and preliminary outline of chemical polluting agents.

\section{Results}

The methodology has been applied in different situations of small and medium craftsmanship
Italian enterprises: leather goods, food, technical dental work, production of artistic ceramics and stained glass, beekeeping activities etc..

Because of space limitations only some of the results will be presented [2,3]. The results are shown in the attached figures in short patterns.

Each of the works studied was evaluated according to the first defined stages

-the key-enters

-the quick assessment of biomechanical overload

-the true risk assessment with the OCRA checklist (upper limb) and RNLE (spine)

Also because of space limitations, we report only the overall risk profiles obtained with the use of the first two stages of evaluation, namely the keyenter and the quick assessment. Histograms that show the overall risk profile of the task are "automatically" obtained through the compilation of software specifically designed

In the field of leather processing, there are different risks in the Tannery (preparation of the skin, Figure 1) rather than in the bag of bags (Figure 2). The application of this methodology has shown a risks of biomechanical overload and of chemical, both with need for an urgent intervention.

Even in the food industry (Figure 3,4) and honey production (Figure 5), risk of biomechanical overload and chemical agents (flour dust in pastry and pizza production and germicides in honey production) are emerged.

The biomechanical overload was also important in the activity of dental technician, with particular attention to repetitive movements of upper limbs (Figure 6). Even in the production of artistic ceramics (Figure 7) and stained glass (Figure 8) the main risks highlighted are both the biomechanical overloads and chemical hazards.

\section{Conclusion}

The proposed methodology, using specific key enters and quick assessment criteria, allows a simple ergonomics hazards identification and risk estimation to be made. It is thus possible to decide, for which occupational hazards, a more exhaustive risk assessment will be necessary and which occupational consultant should be involved (occupational physician, safety engineer, industrial hygienist, etc.).

The presented experiences of pre-mapping occupational hazards and risks (and many others not 
shown here) were performed by different professionals in different working sectors, all over the Italian country.

The analysis methodology, developed in subsequent stages different for depth, and its specific computer support tool, have had a very positive feedback. The analysis system was judged to be very simple to use, and affordable for even non-expert personnel.

This vast experience of national collaboration, made possible (kept by practical suggestions) to improve the proposed tool aimed to map the professional hazards.

We are making concrete the objective of the working group of IEA and of WHO, that is to create simple tools for occupational risk assessment, usable also by non experts.

Considering that the current literature $[5,10,11$, $12,13,14,15,16]$ on the considered topic provides sufficient criteria and methods, our suggestion to other participants in this specific working group, is that what we really need is to turn them into simple and usable tools also applicable to non-experts. To achieve this goal we must begin to produce new patterns testing experimentally on the field. Without these experimental moments, it would still take good reviews of the literature but again only a theoretical contribution.

\section{References}

[1] CEN. EN 1005-2. Safety of machinery - Human physical performance - Part 2:Manual handling of machinery and component parts of machinery. 2003

[2] Colombini D., Occhipinti E., et al: Movimentazione manuale dei carichi: manuale operativo per l'applicazione del Dec.Leg81/08. Dossier Ambiente:
2010;89. primo trimestre.

[3] Colombini D., Di Leone G., Occhipinti E., Montomoli L et al.: Ipotesi di tecniche semplificate per la prima mappatura dei rischi professionali nel settore artigiano. Prima parte: rischi legati all'ergonomia". G Ital Med Lav Erg 2009; 31:3; 292-296

[4] DRAFT CD ISO 12259-2009: Application document guides for the potential users of the ISO 11228-1, 2, 3;- Standards and ISO 11226 on Working Postures

[5] Engkvist Inga-Lill, Hagberg M., Wigaeus-Hjelm Ewa, Menckel et al.. PROSA STUDY GROUP: Interview protocols and ergonomics checklist for analysing overexertion back accidents among nursing personnel. Applied Ergonomics1995, 26-3, 213-220.

[6] ISO 11226. Ergonomics — Evaluation of static working postures. 2000

[7] ISO 11228-1. Ergonomics - Manual handling - Lifting and carrying -2003

[8] ISO 11228-2. Ergonomics - Manual handling - Pushing and pulling- 2007

[9] ISO 11228-3. Ergonomics - Manual handling - Handling of low loads at high frequency- 2007

[10] Malchaire J., Strategia SOBANE e guida per l'individuazione dei rischi, versione 1,2. 2008. Direction general de humanisation de Travail, Unione Europea.

[11] MINISTÈRE DE L'ÉCONOMIE, DE L'INDUSTRIE ET DE L'EMPLOI Identification $d u$ questionare Republique Francaise SUMER 2009.

[12] NIOSH Checklist per l'analisi generale dei rischi ergonomici; www.societadiergonomia.it/download/down/90.doc

[13] OSHA Ergonomics checklist: progetto per gli standard ergonomici OSHA. checklist per la valutazione del luogo di lavoro; www.societadiergonomia.it/download/down/89.doc

[14] OHSW PROCEDURE: OHSW16. Workplace inspectionGeneral Environment. Workplace; www.unisa.edu.au/ohsw/forms/docs/OHSW16.doc

[15] OHSW PROCEDURE: OHSW41. Plant Hazard Identification \& Risk Assessment. Workplace. www.unisa.edu.au/ohsw/forms/docs/OHSW41

[16] REXROTH BOSCH GROUP: La check-list dell'ergonomia per i sistemi di lavoro manuali - Una guida alla creazione di posti di lavoro ergonomici; www.fsm.it/gimle/31/3/10 
Figure 1

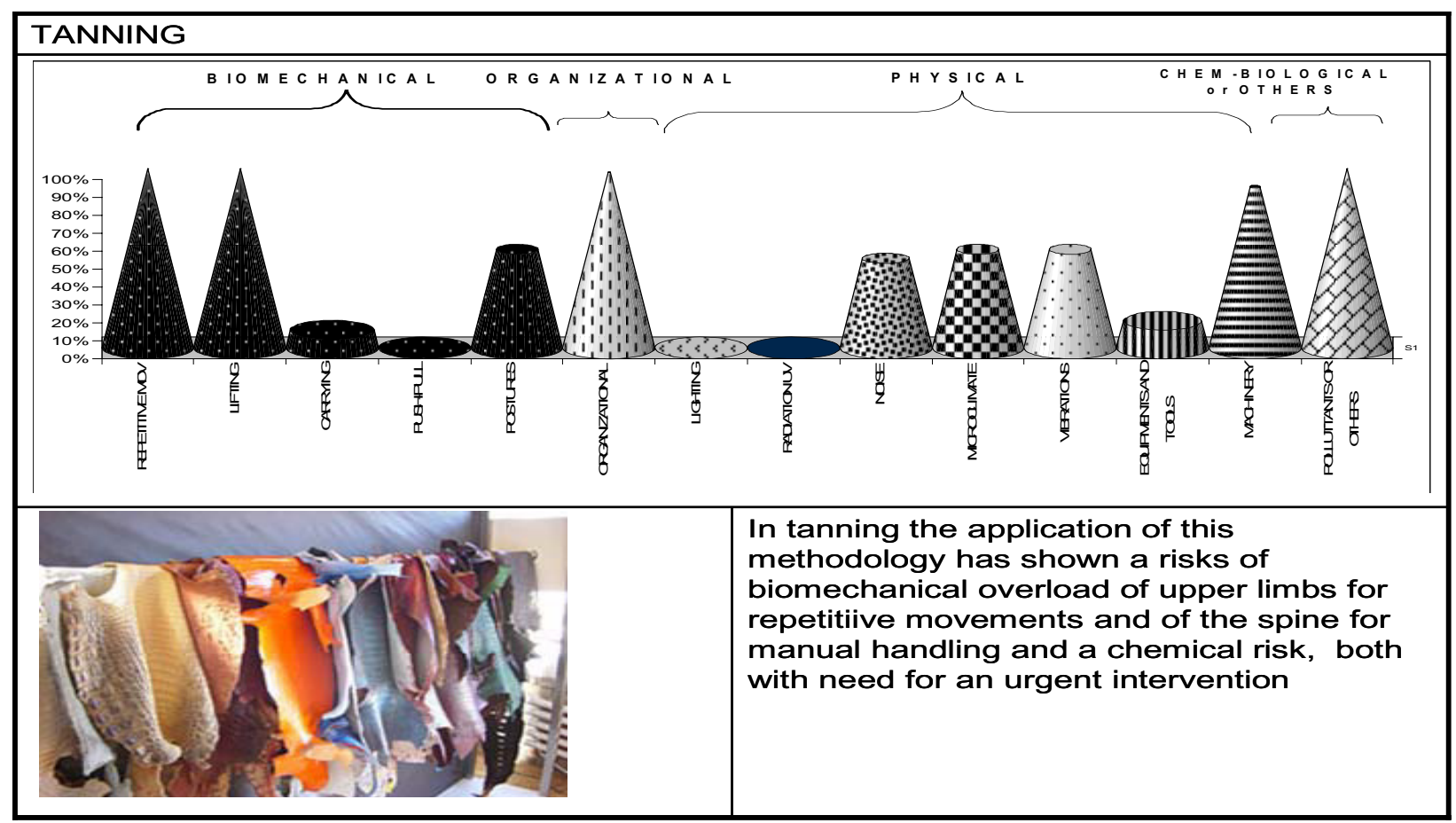

Figure 2

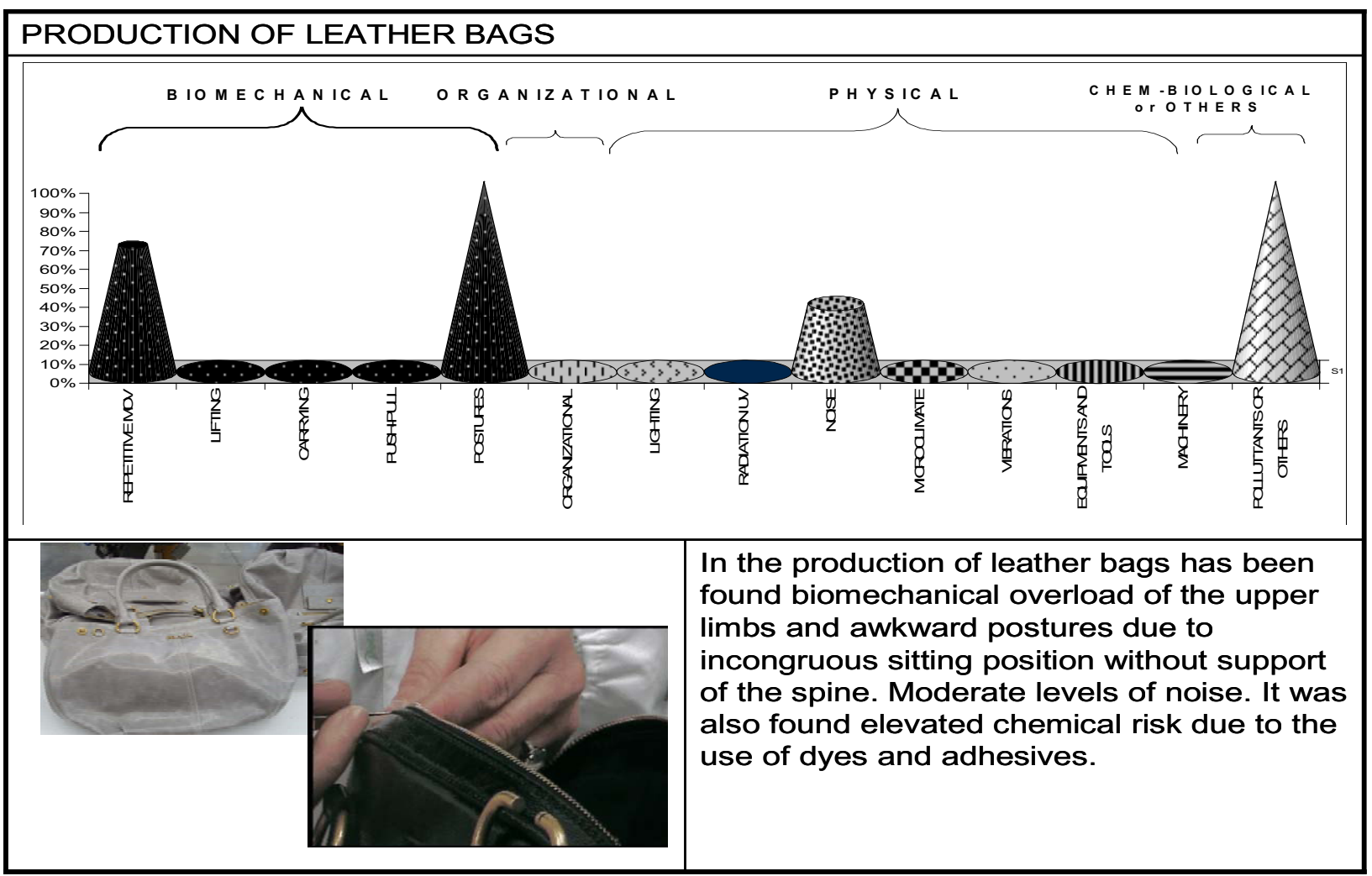


Figure 3

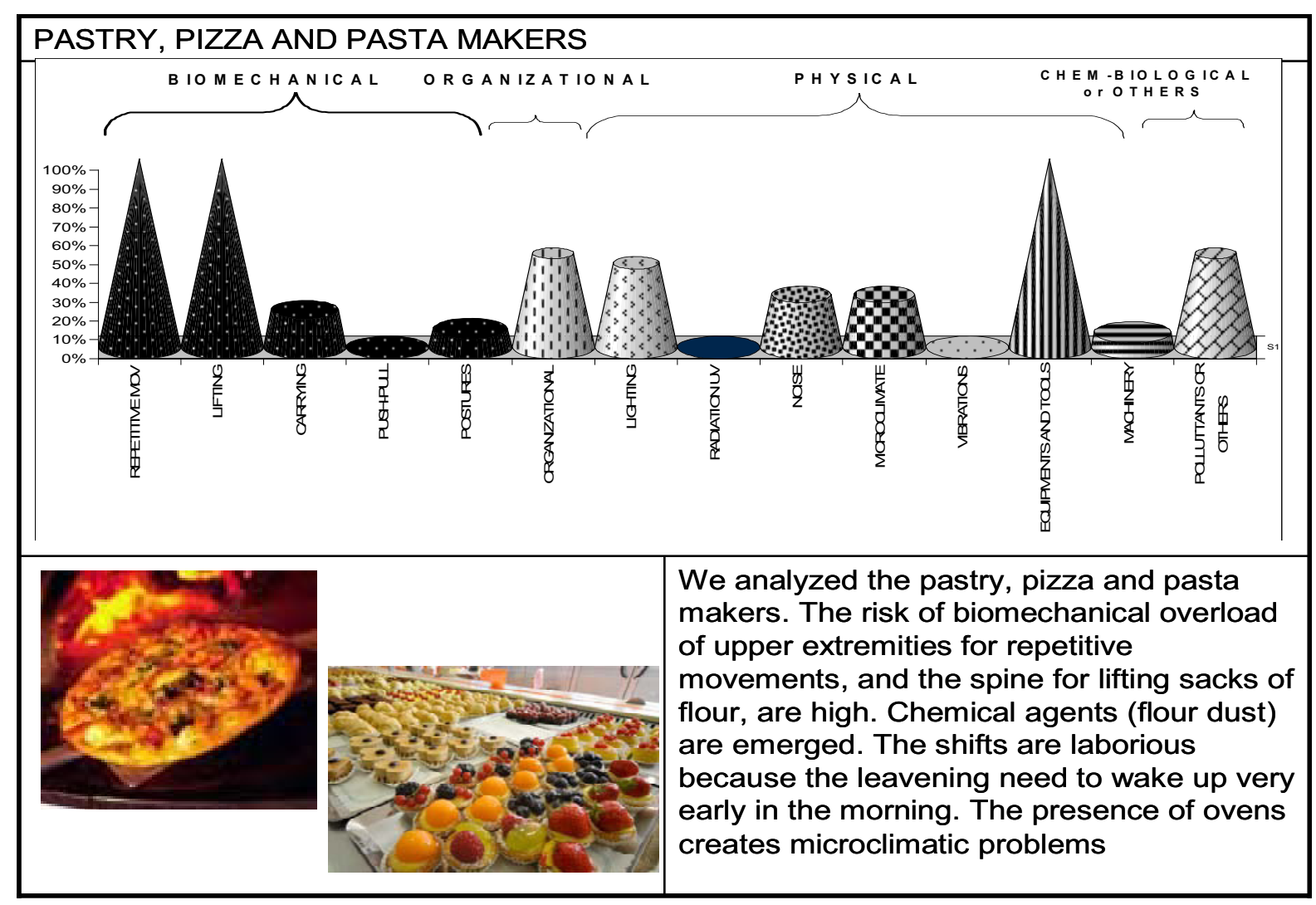

Figure 4




Figure 5

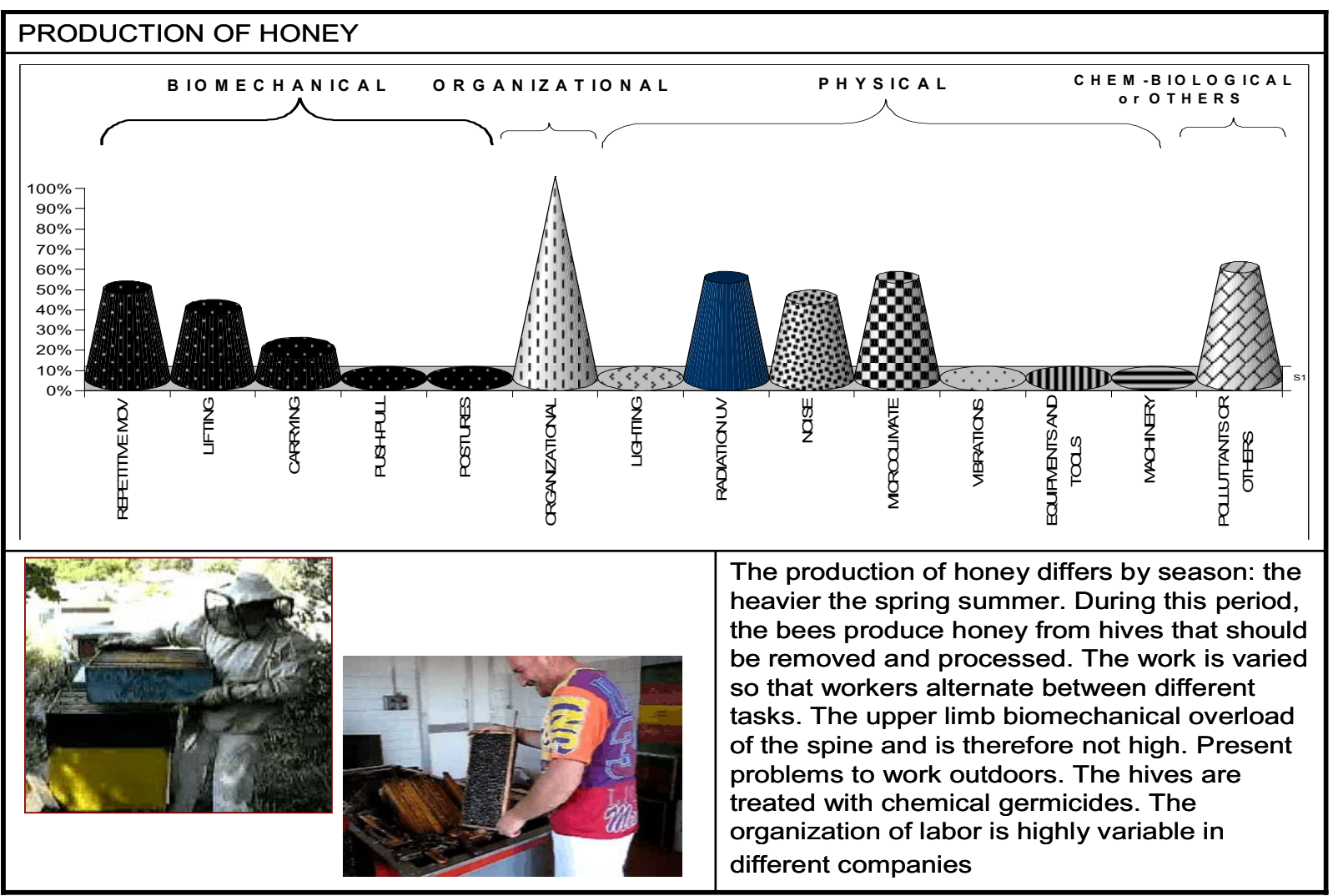

Figure 6

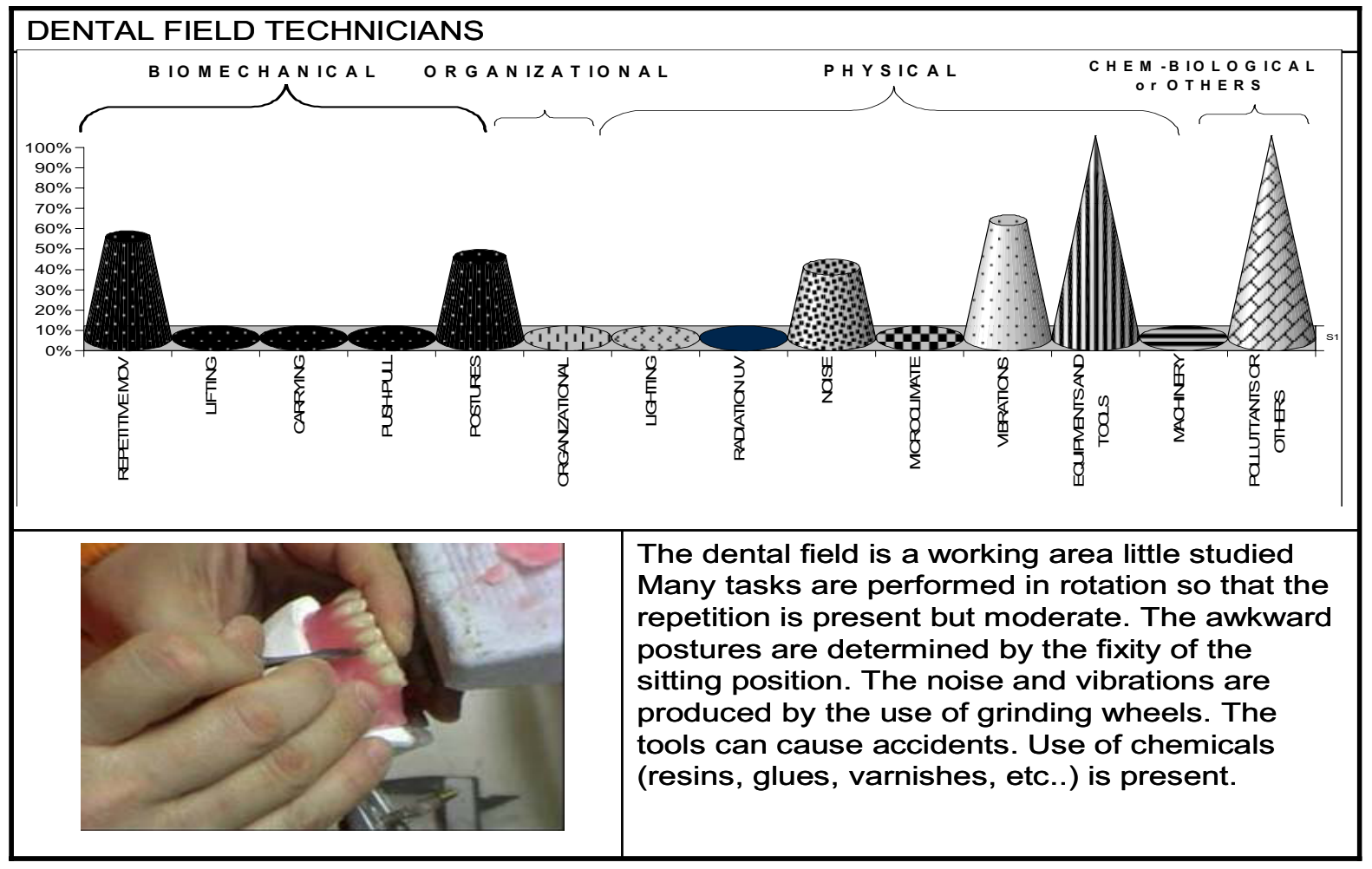


Figure 7

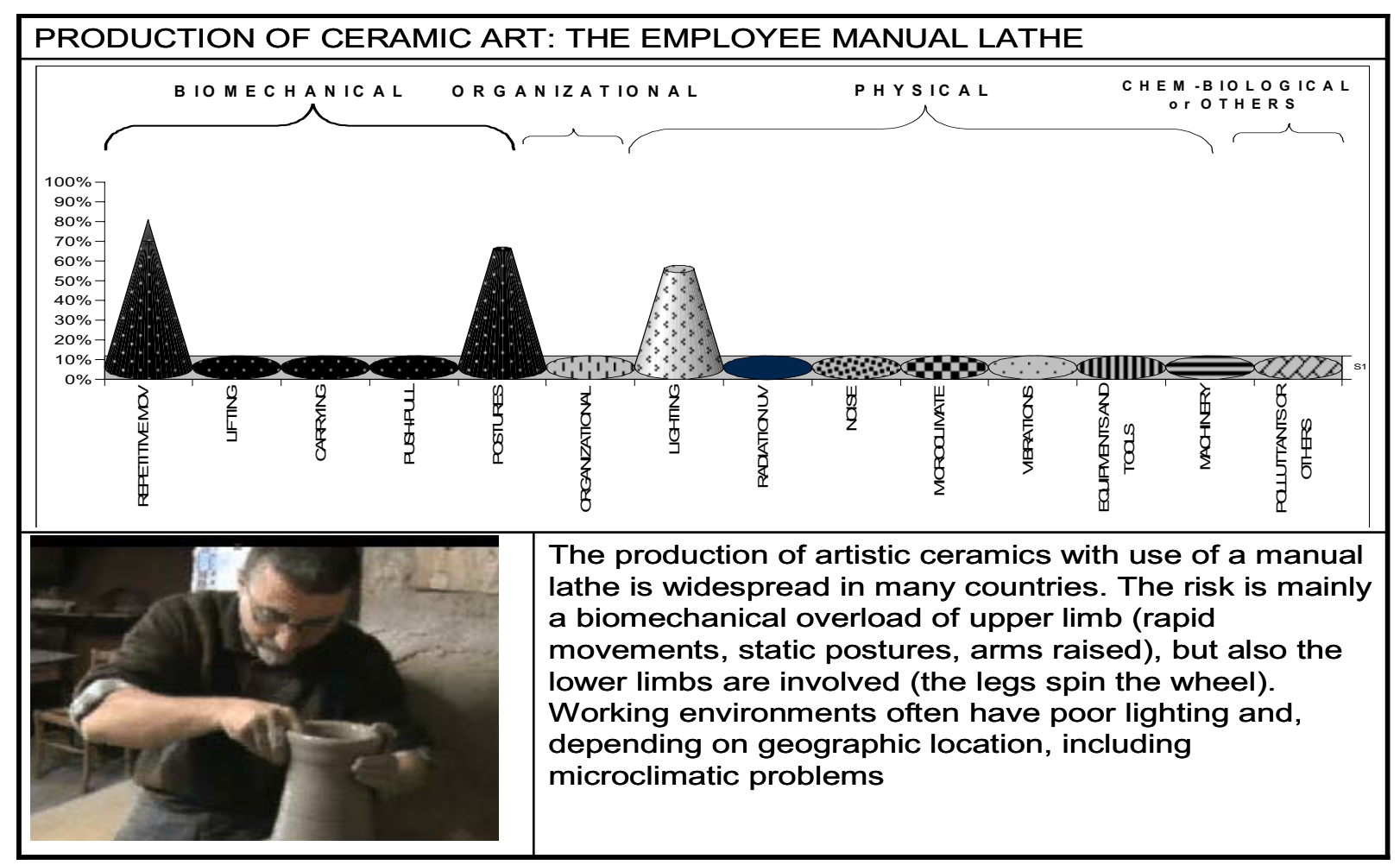

Figure 8

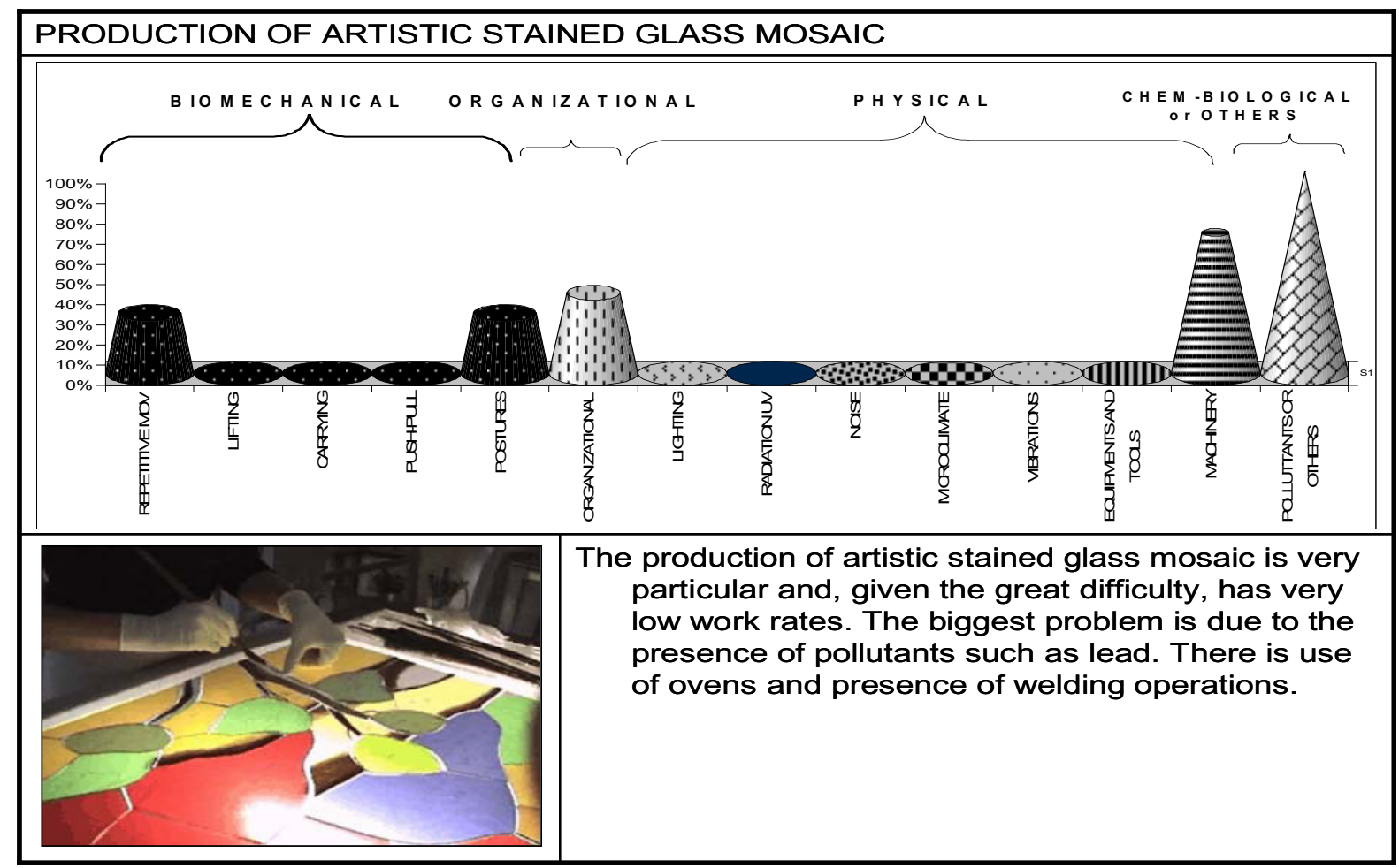

